\title{
Prediction of drug-related problems in diabetic outpatients in a number of hospitals, using a modeling approach
}

This article was published in the following Dove Press journal:

Drug, Healthcare and Patient Safety

28 July 2017

Number of times this article has been viewed

\author{
Ghaith M Al-Taani' \\ Sayer I Al-Azzam² \\ Karem H Alzoubi ${ }^{2}$ \\ Feras W Darwish Elhajji ${ }^{3}$ \\ Michael G Scott ${ }^{4}$ \\ Hamzah Alfahel ${ }^{5}$ \\ Mamoon A Aldeyab ${ }^{6}$ \\ 'Department of Pharmacy Practice, \\ Faculty of Pharmacy, Yarmouk \\ University, ${ }^{2}$ Department of Clinical \\ Pharmacy, Faculty of Pharmacy, \\ Jordan University of Science and \\ Technology, Irbid, ${ }^{3}$ Department of \\ Clinical Pharmacy and Therapeutics, \\ Faculty of Pharmacy, Applied Science \\ Private University, Amman, Jordan; \\ ${ }^{4}$ Medicines Optimisation Innovation \\ Centre, Pharmacy and Medicines \\ Management Centre, Northern Health \\ and Social Care Trust, Ballymena, \\ Northern Ireland, UK; ${ }^{5}$ General and \\ Specialized Surgery Department, \\ Faculty of Medicine, Hashemite \\ University, Zarqa, Jordan; ${ }^{6} \mathrm{~S}$ chool of \\ Pharmacy and Pharmaceutical Science, \\ University of Ulster, Coleraine, \\ County Londonderry, UK
}

Objective: Drug-related problems (DRPs) are considered a serious, expensive, and important undesirable complication of health care. However, as current health care resources are limited, pharmacist DRP services cannot be provided to all patients. Using a modeling approach, we aimed to identify risk factors for DRPs so that patients for DRP-reduction services can be better identified.

Methods: Patients with diabetes from outpatient clinics from five key university-affiliated and public hospitals in Jordan were assessed for DRPs (drug without an indication, untreated indication, and drug efficacy problems). Potential risk factors for DRPs were assessed. A logistic regression model was used to identify risk factors using a randomly selected, independent, nonoverlapping development (75\%) subsample from full dataset. The remaining validation subsample (25\%) was reserved to assess the discriminative ability of the model.

Results: A total of 1,494 patients were recruited. Of them, $81.2 \%$ had at least one DRP. Using the development subsample ( $n=1,085)$, independent risk factors for DRPs identified were male gender, number of medications, prescribed gastrointestinal medication, and nonadherence to self-care and non-pharmacological recommendations. Validation results $(n=403)$ showed an area under the receiver operating characteristic curve of $0.679(95 \%$ confidence interval $=0.629-0.720)$; the model sensitivity and specificity values were $65.4 \%$ and $63.0 \%$, respectively.

Conclusion: Within the outpatient setting, the results of this study predicted DRPs with acceptable accuracy and validity. Such an approach will help in identifying patients needing pharmacist DRP services, which is an important first step in appropriate intervention to address DRPs.

Keywords: medication-related problems, drug-related problems, pharmaceutical care, outpatient, diabetes

\section{Introduction}

The concept of drug-related problems (DRPs) was proposed with the launch of the new cognitive pharmacist service model of pharmaceutical care in the 1990s. DRPs can result in the nonattainment of therapeutic outcomes relevant to the patient's condition or patient harm (e.g., an adverse effect). ${ }^{1}$ DRPs are common within the diabetic population. In general, DRPs are highly undesirable and associated with increased utilization of health care resources, therapeutic failure, or the development of new problems. $^{2-6}$ In addition, increased number of DRPs is a global problem. ${ }^{6-8}$ In patients with diabetes, DRPs are usually associated with polypharmacy, multicomorbidity, and increasing age. ${ }^{6,8}, 9$ Other factors in patients with diabetes that may increase the risk of DRPs are renal impairment, poor lipid control, cardiovascular disease, and the duration of hospital stay, if hospitalized. ${ }^{6,8}$ In general, risk factors for DRPs may also include
Correspondence: Ghaith M Al-Taani Department of Pharmacy Practice, School of Pharmacy, Yarmouk University, PO Box 566, Shafeeq Irshedat Street, Irbid, Jordan

Tel +962272 I IIII

Fax +96227211165

Email galtaani0I@qub.ac.uk 
dementia, poor knowledge by the patient, narrow therapeutic index medication, nonadherence, and specific medications (i.e., antiepileptics, anticoagulants, and insulin). ${ }^{9}$

With the increasing number and complexity of therapeutics, the identification and resolution of DRPs are essential tasks for the pharmacist. Accordingly, efforts to address DRPs have been adopted in a number of pharmaceutical societies and professional bodies internationally. The current financial environment within primary care cannot provide DRP services to all patients. In this study, we aimed to identify the risk factors for DRPs through pharmacist review in outpatient setting in a number of public and university hospitals in Jordan, which can be utilized to identify patients who need DRP services.

\section{Methods}

\section{Setting}

This is a multi-center, cross-sectional study conducted in the outpatient departments of five major hospitals in different geographical locations in Jordan. The following were the study hospitals: King Abdullah University Hospital (KAUH, a teaching hospital affiliated with the Jordan University of Science and Technology in northern Jordan), Jordan University Hospital (a teaching hospital affiliated with the University of Jordan in the central area of Jordan), Al-Basheer Hospital (a public hospital in the central area of Jordan), Princess Basma Hospital (a public hospital in northern Jordan), and Al-Karak Hospital (a public hospital in southern Jordan). Research ethics committees that oversee these hospitals approved the study protocol for the study.

\section{Patients and subjects}

Consecutive patients who attended consultant-led outpatient clinics of cardiology and endocrine specialties who met the selection criteria were invited to participate in this study during the study period (from September 2012 to December 2013). Following were the inclusion criteria: patients with diabetes who are more than 18 years of age and with one or more acute or chronic medical conditions and who have used at least two medications. The study was explained to the patients, and a patient information leaflet was issued to consider participation in this study. Patients who agreed to participate provided written informed consent to participate in this study.

\section{Data collection}

DRPs were identified for the recruited patients. Having a DRP was considered as an outcome variable. Following DRPs were assessed: drugs without an indication, untreated indication, more effective medication available, and treatment needs to be stepped up or down. These were categorized as important DRPs and were included (apart from under-prescribing) in Hanlon's Medication Appropriateness Index (MAI) for the assessment of appropriateness of prescribing with higher weight than other prescribing issues. ${ }^{10}$

Potential variables with the plausibility to be risk factors for DRPs were assessed using a standard statistical analysis as independent predictors for these problems. DRPs were defined according to Hepler and Strand's definition. ${ }^{1}$

Recruited patients were assessed by clinical pharmacists, who hold a Doctor of Pharmacy degree and license to practice pharmacy in the jurisdiction, for the presence of DRPs. For consistent implementation, a systematic procedure was used; clinical pharmacists were trained on the documentation, and the implementation of the procedures was monitored. These assessments utilized a validated pharmaceutical care manual, which included development of patient database that allows identification and classification of DRPs according to AbuRuz et al's classification system. To identify DRPs, an evidence-based approach, which systematically incorporates best current evidence in decision making was used. Patients were interviewed and assessed, and their laboratory data were reviewed for DRPs by referral to medical records. ${ }^{11,12}$ Referral to current evidence-based national and international guidelines and compendium was utilized for the identification of DRPs. For example, the indication of medications included two categories "drug without indication" (unnecessary therapy) and "untreated condition" and was checked using current drug information resources such as the Drug Information Handbook (lexi-comp), in terms of inconsistencies between drug indication and the disease conditions the patient is having. Medication efficacy assessments utilized patients' clinical characteristics and by referral to current evidencebased guidelines. "More effective drug is available" is the case where the drug is indicated for the medical condition but is not the best therapy, according to current treatment guidelines. "The patient requires additional combination therapy or stepping up", which can be recommended by referral to current guidelines or due to failure of the drug to achieve the therapeutic goals, actually or potentially. "The patient treatment should be stepped down", via referral to current guidelines. The DRP classification system used was a modification of Aburuz et al's valid and reliable classification system of DRPs. The latter classification was tested for validity and reliability on cases of 200 patients and shown 
to be internally and externally valid and reproducible and demonstrated good inter-rater agreement. ${ }^{13,14}$

A number of variables collected in the course of study were assessed as potential risk factors for outcomes of DRPs. These variables included age, gender, educational level (illiteracy, school, and university), health insurance, smoking history, number of medications, British National Formulary (BNF) medications categories, knowledge about medication and nonadherence to self-care, and lifestyle recommendations related to the disease.

\section{Data analysis}

Standard statistical methodologies were used in the analysis of risk factors for DRPs. Data were entered into SPSS version 21 and appropriately coded. All study data were split into randomly selected, independent, nonoverlapping development (75\%) and validation (25\%) subsamples.

A logistic regression analysis was used in the assessment of independent risk factors associated with the outcome variable using a predictive approach. The development subsample was used to develop a logistic regression model whereas the validation subsample was reserved for the cross-validation of the analysis. For the development subsample, candidate variables to be included into the model were assessed using a chi-square test and independent samples $t$-test. Candidate variables were defined as having a $p$ value of not more than 0.25 (i.e., having a trend of association with the outcome variable). Candidate variables were then included in a "backward" logistic regression model, which serves to retain factors with an independent, significant contribution to the outcome variable. On the validation subsample, the probability score for each patient record for DRPs was determined using the model equation developed on development subsample data. A receiver operating characteristic (ROC) curve was constructed to compare the DRPs' actual status for the patient with the probability scores achieved using the proposed model. This ROC curve analysis produced a statistic "area under the curve" and significance level relating to the discriminative ability of the model. To be useful as a screening tool threshold or cutoff point for the resultant model. Patients who scored higher than this cutoff point would potentially be at higher risk of DRPs cutoff point would potentially be at higher risk of DRPs. The cutoff point was determined using Youden's index. ${ }^{15}$ Sensitivity (true positive rate) and specificity (true negative rate) were also calculated. Based on the results of sensitivity and specificity analysis, an economic evaluation of the cost saving associated with targeting mechanism was performed. The following parameters were measured: intervention cost, cost of DRPs, and hypothesized ability of the pharmacy to address DRPs.

\section{Results General characteristics}

The total number of recruited patients over the study period was 1,494 . More than three quarters (81.2\%) of the patients had DRPs. In relation to indication-related DRPs, $26.1 \%$ of the recruited patients had a at least one drug without an indication and $19.6 \%$ had one or more untreated conditions. Whereas effectiveness-related DRPs were manifested in $16.7 \%$ of the recruited patients as "more effective drug is available", $19.6 \%$ as "the patient requires additional combination therapy or stepping up", and $10.6 \%$ as "the patient treatment should be stepped down".

The mean age of patients was 58.4 years, with approximately $30 \%$ of the patients being more than 65 years old. The mean number of medications was 6.5 medications per patient.

In this study, the percentage of female patients was higher than that of male patients. Regarding the educational level, about $18 \%$ were illiterate and approximately $60 \%$ and $20 \%$ of the sample received only school education (i.e., primary and secondary education) and tertiary education, respectively. The majority of patients had health insurance. The major BNF medication categories (body system) were cardiovascular, endocrine, and gastrointestinal medications. About half of the patients had a problem with knowledge of their illness. Almost $90 \%$ of the patients were nonadherent to self-care and life-style recommendation related to their disease.

Table 1 summarizes the general characteristics of the recruited patients for the development and validation dataset. We found similar characteristics between development and validation datasets (e.g., $59.8 \%$ and $59.5 \%$ of the patients were females in the development and validation datasets, respectively).

\section{Risk factors for important DRPs}

Using the development subsample data, a logistic regression model was produced to assess potential risk factors associated with the presence of DRPs. Table 2 summarizes the independent risk factors for DRPs using a logistic regression analysis identified together with their odd ratios.

To validate the analysis and ensure generalizability, the model developed using the development subsample data was applied in the validation dataset to assess model discrimination between the DRPs from the remainder of the patients. An ROC curve was constructed. Statistical significance $(p \leq 0.001)$ for discriminative ability was achieved for both 
Table I Development and validation datasets characteristics

\begin{tabular}{|c|c|c|c|}
\hline Variable & & Development $(n=I, 085 ; \%)$ & Validation $(n=403 ; \%)$ \\
\hline \multirow[t]{3}{*}{$\overline{\text { Age }}$} & Adults & 69.3 & 70.7 \\
\hline & Elderly & 30.7 & 29.3 \\
\hline & Missing & 0.5 & \\
\hline \multirow[t]{3}{*}{ Gender } & Male & 40.2 & 40.5 \\
\hline & Female & 59.8 & 59.5 \\
\hline & Missing & 0.5 & 0.2 \\
\hline \multirow[t]{4}{*}{ Educational level } & Illiterate & 17.9 & 18.4 \\
\hline & School & 60.7 & 59.3 \\
\hline & University & 21.4 & 22.3 \\
\hline & Missing & 0.2 & \\
\hline \multirow[t]{3}{*}{ Health insurance } & Insured & 97.3 & 96.3 \\
\hline & Uninsured & 2.7 & 3.7 \\
\hline & Missing & 0.4 & \\
\hline \multirow[t]{12}{*}{ Medication (organ system BNF) } & Gastrointestinal & 32.8 & 33.7 \\
\hline & Cardiovascular & 89.6 & 88.1 \\
\hline & Respiratory & 7.9 & 9.9 \\
\hline & CNS & 16.9 & 19.9 \\
\hline & Infections & 0.8 & 0.5 \\
\hline & Endocrine & 79.3 & 82.4 \\
\hline & Genitourinary & 3.0 & 3.0 \\
\hline & Malignancy and immunosuppressants & 0.6 & 0.2 \\
\hline & Nutrition and blood & 19.3 & 17.1 \\
\hline & Musculoskeletal & 9.4 & 8.9 \\
\hline & Eye, ear, nose, and oropharynx & 0.2 & 0.2 \\
\hline & Skin & 0.1 & 0.0 \\
\hline Problem in illness knowledge & & 46.6 & 46.7 \\
\hline Nonadherence to self-care & & 91.9 & 89.8 \\
\hline
\end{tabular}

Abbreviations: BNF, British National Formulary; CNS, central nervous system.

Table 2 Odd ratios for the variables associated with important DRPs

\begin{tabular}{lllll}
\hline Variable & $\boldsymbol{\beta}(\mathrm{SE})$ & Odds ratio & $\mathbf{9 5 \%} \mathbf{C l}$ for odds ratio & $\boldsymbol{p}$-value \\
\hline Number of medications & $0.129(0.033)$ & 1.137 & $1.065-1.214$ & $<0.001$ \\
Male gender & $0.467(0.172)$ & 1.595 & $1.138-2.236$ & 0.007 \\
Nonadherence to self-care & $0.695(0.257)$ & 2.004 & $1.211-3.316$ & 0.007 \\
Gastrointestinal medication & $1.179(0.237)$ & 3.252 & $2.043-5.177$ & $<0.001$ \\
Constant & $-0.395(0.299)$ & 0.674 & & 0.188 \\
\hline
\end{tabular}

Abbreviation: DRPs, drug-related problems.

the development and validation dataset. Area under the ROC curve achieved for the development dataset was 0.702 (95\% confidence interval equaled $0.665-0.740$ ), whereas area under the ROC curve achieved for the independent, nonoverlapping, random cross validation subsample was 0.679 (95\% confidence interval equaled 0.629-0.720). Figure 1 illustrates the ROC curve for this model on the validation subsample.

To assess the utility of the model as a screening tool for DRPs, a cutoff point was identified for the resultant model's predicted probabilities. Patients who scored higher than this threshold were considered being at increased risk of DRPs. This cutoff score was the predicted probability that equaled 0.76 . The sensitivity and specificity for this cutoff score equaled $65.4 \%$ and $63.0 \%$, respectively, indicating that two-thirds of the patients who have DRPs are correctly identified, with almost the same percentage of patients not having DRPs being correctly classified as being not at risk of DRPs.

\section{Discussion}

This study identified the risk factors for DRPs, which were used to predict these outcomes with appropriate discrimination, sensitivity, and specificity. Such an approach could prove useful in identifying patients for DRPs services. The impact of this cannot be underestimated, given that pharmacist services are provided to patients at risk of DRPs. Assuming an intervention cost of $\$ 50$ and average cost of DRPs of $\$ 875$ (as derived from Ernst and Grizzle publication), ${ }^{3}$ if the pharmacist intervention reduced the DRPs' rate by $40 \%$, using the modeling approach, 


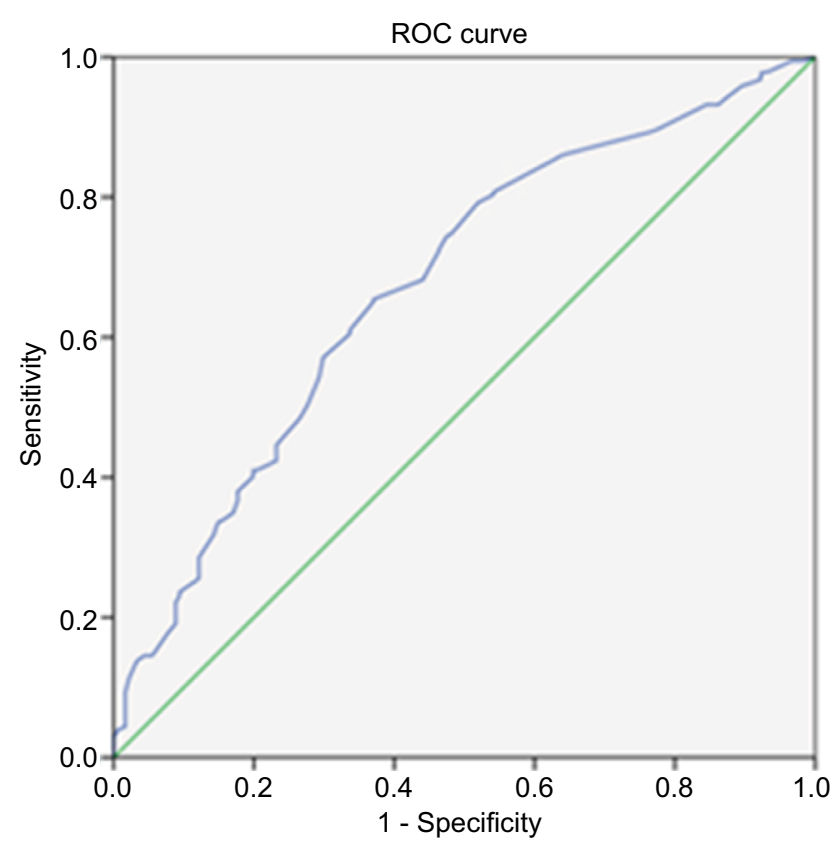

Figure I ROC curve for the logistic regression model for DRPs (validation subsample).

Abbreviations: DRPs, drug-related problems; ROC, receiver operating characteristic.

the cost of the DRPs would be $\$ 577,175$, whereas the cost associated without the modeling approach would be $\$ 762,125$. The ratio for cost-savings was $24.3 \%$. This high-level of costsavings could realize benefits for the health care system as well as patients. Such benefits can be a golden opportunity for pharmacists to improve the care they provide to patients. So far, this is the first study performed to predict DRPs for outpatient diabetics in Jordan using a modeling approach, which is considered superior to other approaches that address the inherent limitations of other methods. ${ }^{16}$

Almost all recruited patients had at least one DRP. About $80 \%$ of the patients had a DRP related to the indication and effectiveness of therapy. This indicates that the patients in the study sample were high-risk patients, and such frequent problems threaten patient safety. This is a health challenge that can be addressed by pharmacists. In two studies, most of the patients $(>80 \%)$ had at least one DRP, which is consistent with our analysis. ${ }^{6,8}$ Despite the presence of two teaching, university-affiliated hospitals among the study hospitals, such alarming numbers of DRPs in this study population highlights a drawback of the medication management for those patients.

Surprisingly, only $30 \%$ of the patients were more than 65 years old, indicating that the patient population is young, which makes it strange to have a high number of DRPs. It is well known that polypharmacy and multi-comorbidity of patients becomes more prevalent with increasing age, thereby increasing the propensity for DRPs. In light of this phenomenon, one study that assessed DRPs in patients with diabetes reported about $15 \%$ more nonelderly patients than elderly patient in the study population. ${ }^{8}$

The most common medication groups were endocrine and cardiovascular medications. An indicator for poor care quality identified in this study is poor illness knowledge, with about half of the patients having a problem with illness knowledge. This poses questions as to whether health care professionals are doing what is needed to support patients in coping with and understanding their illness, particularly in patients with diabetes.

Studies extensively have reported that the increased number of medication and increased age are considered important risk factors for DRPs, adherence problems, adverse effects, and drug interactions. ${ }^{8,17-24}$ This study highlighted that having an increased number of medications and being in the elderly group ( $>65$ years old) are independent predictors for DRPs. This can be related to polypharmacy, multi-comorbidity, and decreased brain function with increased age. These identified factors can make medication management difficult for the patient and the health care professional.

Misbehavior by patients manifested as nonadherence to self-care and nonpharmacological recommendations was prevalent in patients and a predictor for DRPs. Pharmacists and other health care professionals shall emphasize the importance of adherence and highlight the negative consequences of nonadherence. As an independent predictor of DRPs, nonadherence is in line with what is hypothesized. A Danish study highlighted that an inappropriate lifestyle is a common type of DRP in patients with diabetes. ${ }^{7}$ Based on a literature review and expert opinion, a previous study highlighted that nonadherence is an important risk factor for DRPs. ${ }^{9}$

Male gender as an independent predictor for DRPs can be related either to biological differences or differences in health behavior between males and females. Health behavior can differ between males and females, although not many studies support this notion. Female patients might attend clinic visits more often than male patients and thus be more likely to comply with drug therapy and with dietary recommendations. ${ }^{25,26}$ In one study, male gender was identified as a risk factor for DRPs in patients with diabetes, ${ }^{6}$ but little is known about the role of biological differences between males and females.

\section{Conclusion}

Due to the increased number of DRPs in this study's population, we recommend that an increased intensity of pharmacist services be provided to patients; indeed, the 
better identification mechanism presented in this study will pave the way for such improved patient-centered services directed against DRPs in patients with diabetes. The modeling approach is considered the first step in designing patientcentered services to address DRPs. In developing countries like Jordan, guidelines for best practices internationally can be adapted to fit the local health situation. Both community and hospital pharmacists should take part in addressing DRPs in patients with diabetes, as the DRPs are prevalent and the patients are considered at high risk for DRPs. A simplistic approach that can be applied in routine practice is that pharmacists need to take extra care when dealing with patients with diabetes to check for DRPs; in this regard, pharmacists can follow a simple checklist to identify and resolve DRPs.

\section{Acknowledgment}

This project was supported by the Scientific Research Funds (project number: 104/2012) at the Ministry of Higher Education and Scientific Research, Amman, Jordan.

\section{Disclosure}

The authors report no conflicts of interest in this work.

\section{References}

1. Hepler CD, Strand LM. Opportunities and responsibilities in pharmaceutical care. Am J Hosp Pharm. 1990;47(3):533-543.

2. Johnson JA, Bootman JL. Drug-related morbidity and mortality. A cost-of-illness model. Arch Intern Med. 1995;155(18):1949-1956.

3. Ernst FR, Grizzle AJ. Drug-related morbidity and mortality: updating the cost-of-illness model. JAm Pharm Assoc (Wash). 2001;41(2):192-199.

4. Hartholt KA, van der Velde N, Looman CW, et al. Adverse drug reactions related hospital admissions in persons aged 60 years and over, The Netherlands, 1981-2007: less rapid increase, different drugs. PLoS One. 2010;5(11):e13977.

5. Sarkar U, López A, Maselli JH, Gonzales R. Adverse drug events in U.S. adult ambulatory medical care. Health Serv Res. 2011;46(5):1517-1533.

6. Zaman Huri H, Chai Ling L. Drug-related problems in type 2 diabetes mellitus patients with dyslipidemia. BMC Public Health. 2013;13:1192.

7. Haugbølle LS, Sørensen EW. Drug-related problems in patients with angina pectoris, type 2 diabetes and asthma-interviewing patients at home. Pharm World Sci. 2006;28(4):239-247.

8. Zaman Huri H, Fun Wee H. Drug related problems in type 2 diabetes patients with hypertension: a cross-sectional retrospective study. $B M C$ Endocr Disord. 2013;13:2.
9. Kaufmann CP, Stämpfli D, Hersberger KE, Lampert ML. Determination of risk factors for drug-related problems: a multidisciplinary triangulation process. BMJ Open. 2015;5(3):e006376.

10. Hanlon JT, Schmader KE, Samsa GP, et al. A method for assessing drug therapy appropriateness. J Clin Epidemiol. 1992;45(10):1045-1051.

11. AbuRuz SM, Bulatova NR, Yousef AM. Validation of a comprehensive classification tool for treatment-related problems. Pharm World Sci. 2006;28(4):222-232.

12. Aburuz S. The case for evidence-based pharmaceutical care. Res Social Adm Pharm. 2015;11(3):e146-e147.

13. Aburuz SM, Bulatova NR, Yousef AM, Al-Ghazawi MA, Alawwa IA, Al-Saleh A. Comprehensive assessment of treatment related problems in hospitalized medicine patients in Jordan. Int J Clin Pharm. 2011;33(3):501-511.

14. Cipolle RJ, Strand L, Morley P. Pharmaceutical Care Practice: The Clinician's Guide. 2nd ed. New York: McGraw-Hill; 2004.

15. Ruopp MD, Perkins NJ, Whitcomb BW, Schisterman EF. Youden Index and optimal cut-point estimated from observations affected by a lower limit of detection. Biom J. 2008;50(3):419-430.

16. Billings J, Blunt I, Steventon A, Georghiou T, Lewis G, Bardsley M. Development of a predictive model to identify inpatients at risk of re-admission within 30 days of discharge (PARR-30). BMJ Open. 2012;2(4). pii:e001667.

17. Koh Y, Kutty FB, Li SC. Drug-related problems in hospitalized patients on polypharmacy: the influence of age and gender. Ther Clin Risk Manag. 2005;1(1):39-48.

18. Bain KT, Weschules DJ, Tillotson P. Prevalence and predictors of medication-related problems. Medicare Patient Management. 2006;1: $14-26$.

19. Viktil KK, Blix HS, MogerTA, Reikvam A. Polypharmacy as commonly defined is an indicator of limited value in the assessment of drug-related problems. Br J Clin Pharmacol. 2006;63(2):187-195.

20. Alkema GE, Wilber KH, Simmons WJ, Enguidanos SM, Frey D. Prevalence of potential medication problems among dually eligible older adults in Medicaid waiver services. Ann Pharmacother. 2007;41(12): 1971-1978.

21. Rutledge JC, Ng KF, Aung HH, Wilson DW. Role of triglyceride-rich lipoproteins in diabetic nephropathy. Nat Rev Nephrol. 2010;6(6): 361-370.

22. Khdour MR, Jarab AS, Adas HO, Samaro EZ, Mukattash TL, Hallak HO. Identification of drug-related problems: a prospective study in two general hospitals. Curr Clin Pharmacol. 2012;7(4):276-281.

23. Ahmad A, Mast MR, Nijpels G, Elders PJ, Dekker JM, Hugtenburg JG. Identification of drug-related problems of elderly patients discharged from hospital. Patient Prefer Adherence. 2014;8:155-165.

24. Snyder ME, Frail CK, Jaynes H, Pater KS, Zillich AJ. Predictors of medication-related problems among medicaid patients participating in a pharmacist-provided telephonic medication therapy management program. Pharmacotherapy. 2014;34(10):1022-1032.

25. Babwah F, Baksh S, Blake L, et al. The role of gender in compliance and attendance at an outpatient clinic for type 2 diabetes mellitus in Trinidad. Rev Panam Salud Publica. 2006;19(2):79-84.

26. Vlassoff C. Gender differences in determinants and consequences of health and illness. J Health Pop Nutr. 2007;25(1):47-61.
Drug, Healthcare and Patient Safety

Publish your work in this journal

Drug, Healthcare and Patient Safety is an international, peer-reviewed open access journal exploring patient safety issues in the healthcare continuum from diagnostic and screening interventions through to treatment, drug therapy and surgery. The journal is characterized by the rapid reporting of reviews, original research, clinical, epidemiological and

\section{Dovepress}

post-marketing surveillance studies, risk management, health literacy and educational programs across all areas of healthcare delivery. The manuscript management system is completely online and includes a very quick and fair peer-review system. Visit http://www.dovepress.com/ testimonials.php to read real quotes from published authors. 